\title{
Estimation of the Effective Thermal Properties in a Metallic Medium by an Inverse Technique using Temperatures Measurements
}

\author{
A. Sakly ${ }^{1 \dagger}$, A. Jemni ${ }^{1}$, P. Lagonotte ${ }^{2}$ and D. Petit ${ }^{2}$ \\ ${ }^{1}$ Laboratoire d'Etudes des Systèmes Thermiques et Energétiques, Ecole Nationale d'Ingénieurs de Monastir, Avenue \\ Ibn El Jazzar, Monastir 5019, Tunisie \\ ${ }^{2}$ Laboratoire d'Etudes Thermiques, UMR-CNRS 6608, Ecole Nationale Supérieure de Mécanique et \\ d'Aérotechnique, BP 109, 86960 Futuroscope, France \\ $\dagger$ †orresponding Author Email: ahlemsakly@yahoo.fr
}

(Received April 2, 2009; accepted April 12, 2010)

\begin{abstract}
In order to master the use of electric machines and to minimize its thermal losses, the knowledge of thermo-physical properties of metallic materials that constitute them, is important. This study deals with the estimation of several thermal properties in a metallic medium. The system under investigation is a rectangular metallic plate, which is submitted to an homogenous heat power on the volume sample. The direct problem simulates numerically the system and the experimental conditions. An iterative procedure, based on minimizing a sum of squares norm with the Levenberg-Marquardt method, is used to solve the inverse problem. In order to characterize the thermal behavior of metallic materials, an experimental set-up was built. The measured temperature data using infrared camera are used to estimate the effective thermal conductivity, the effective volumetric heat capacity as well as the global heat transfer coefficient with the environment.
\end{abstract}

Keywords: Thermal properties, Numerical simulation, Experimental characterization.

\section{NOMENCLATURE}

$\begin{array}{ll}c_{p} & \text { specific heat capacity }\left(\mathrm{J} \mathrm{kg}^{-1} \mathrm{~K}^{-1}\right) \\ e & \text { sample thickness }(\mathrm{m}) \\ h & \text { global heat transfer coefficient }\left(\mathrm{W} \mathrm{K}^{-1} \mathrm{~m}^{-2}\right) \\ J & \text { ordinary least-squares norm } \\ l & \text { sample width }(\mathrm{m}) \\ L & \text { sample length }(\mathrm{m}) \\ n & \text { number of measurement points } \\ p & \text { number of unknown parameters } \\ r & \text { number of transient temperature } \\ S & \text { heat power }\left(\mathrm{W} \mathrm{m} \mathrm{m}^{-3}\right)\end{array}$

\section{INTRODUCTION}

The prediction of thermal dissipations in electrical machines involves, while modeling magnetic materials, separation of iron losses into three contributions: static, classical and excess losses (Bertotti1988).

Starting from these considerations, it becomes essential to predict temperature effect on the magnetic losses. Some published works interest to study the influence of temperature on coercive field describing thermal behavior of magnetic hysteresis. Some proposed models allowed the determination of thermal effect on

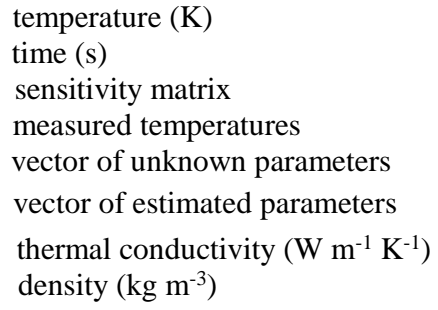

hysteresis characteristics and then on corresponding losses (Féliachi et al. 1999).

The modeling of heat conduction problems, even for complex geometry, has become relatively easy with modern software. Nevertheless, for many industrial problems, it is often difficult to know the thermophysical properties. Estimation of the effective thermal conductivity, the specific capacity and the heat transfer coefficient with the environment is essential for thermal design and numerical simulation of magnetic losses. In 
the past decades, many methods have been presented to determine these properties. Some published works are related to the experimental as well as the theoretical determination of the thermal conductivity and the heat capacity (Jarny et al. 1995). The development of the inverse problem has progressed. Its resolution permits the determination of more than one thermo-physical property and then to characterize complex materials.

In order to study the magneto-thermal coupling in the magnetic materials, a characterization system was built in the LET $^{2}$ to demonstrate the feasibility of the parameters estimation and to characterize metallic materials. In this paper, we propose to estimate the effective thermal properties in a metallic sample (semi process $\mathrm{Fe}-\mathrm{Si}$ ). In our study, the inverse problem is solved to determine three parameters using temperature measurement data from an infrared camera. First, a numerical simulation is given to describe the experience (direct problem). This model is used to show the possibility to estimate simultaneously the thermal properties and to study the impact of measurement noise on the identification accuracy. The estimation is based on minimizing the least squares norm using the Levenberg-Marquardt iterative procedure (inverse problem). Afterwards, an inversion with experimental data obtained with the experimental set-up, is performed and discussed.

\section{Direct Problem}

The system under investigation is a rectangular metallic plate, having a uniform section. In order to limit the thermal study to the plate, we choose to have imposed temperature at the medium boundaries. This physical device exchanges heat through its lateral faces. This plate is submitted to an homogenous power heat.

The heat transfer in the plate is assumed to be twodimensional. The metallic medium $(\mathrm{Fe}-\mathrm{Si})$ is considered homogeneous, and having constant thermal properties and density. Figure 1 shows the plate with the thermal boundaries conditions.

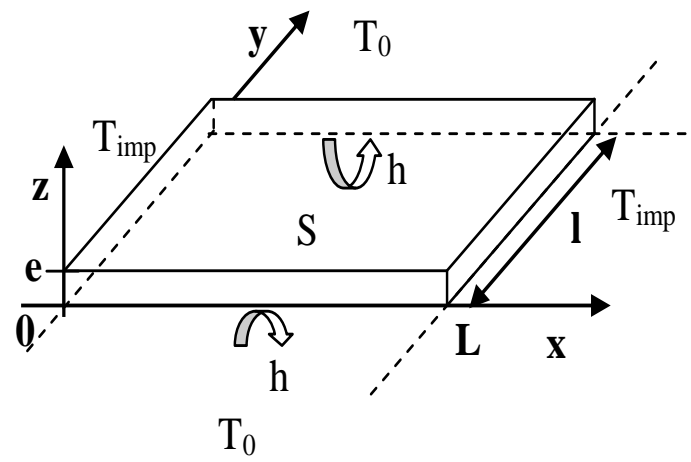

Fig.1. The plate with the thermal boundaries conditions

\subsection{Energy Equation}

The transient temperature distribution inside the plate is described by the two-dimensional heat conduction equation:

$\rho C p \frac{\partial T}{\partial t}=\operatorname{div}(\lambda \overrightarrow{\operatorname{grad} T})+\dot{S}-\frac{2 h}{e}\left(T-T_{0}\right)$
Where $\dot{S}$ is the homogenous heat power, respectively, $\left(\rho c_{p}\right)$ is the heat capacity, $\lambda$ is the thermal conductivity and $\mathrm{h}$ is the global heat transfer coefficient (convection + radiance).

\subsection{Initial and Boundaries Thermal Conditions}

The heat flux continuity through the lateral faces $(y=0$ and $\mathrm{y}=1$ ) allows us to write the following equations:

$$
\begin{gathered}
\lambda \frac{\partial T}{\partial y}=h\left(T(x, 0, t)-T_{0}\right) \\
-\lambda \frac{\partial T}{\partial y}=h\left(T(x, l, t)-T_{0}\right)
\end{gathered}
$$

Initially, we suppose that the temperature in the medium is constant and is equal to the ambient temperature:

$T(0, x, y)=T_{0}$

The system of the presented equations is solved at each time step by the control-volume based finite element method (Patankar et al. 1983). This method leads to the determination of the temperatures at all the grid points. The main advantage of this method is to allow a big flexibility in the considered geometry. It ensures, also, the flux conservation and avoids the generation of parasite sources. Thus, the use of such method will permit to treat complex geometries. A regular mesh within the integrated domain was used. The total number of the grid was $40 \times 32$. The equations are then integrated on the control volume in the time interval $[\mathrm{t}, \mathrm{t}+\Delta \mathrm{t}]$.

\section{INVERSE Problem}

\subsection{Estimation Method}

For the inverse problem considered in this study, the effective thermal conductivity $\lambda$, the effective volumetric heat capacity $\rho c_{p}$, and the global heat transfer coefficient $h$ between the plate and the environment are regarded as unknown. While the other quantities appearing in the formulation of the direct problem described in Eq. (1) are assumed to be known.

The estimation is based on minimizing the ordinary least squares norm (Beck 1969):

$J=\sum_{i=1}^{n}\left[\sum_{j=1}^{r}\left(Y_{i}^{j}-Y_{\bmod , i}^{j}(\beta)\right)^{2}\right]$

Where $\mathrm{n}$ is the number of temperature sensors, $\mathrm{r}$ the number of transient temperatures, $Y_{i}^{j}$ the measured temperature at time $\mathrm{j}$ and point $\mathrm{i}$, and $Y_{\bmod , i}^{j}(\beta)$ the calculated temperature for the same location and time obtained from the solution of the direct problem, by using the current available estimate for the vector of unknown parameters $\beta^{T}=\left[\lambda,\left(\rho c_{p}\right), h\right]$.

$\mathrm{J}$ is optimal if:

$\operatorname{grad} J(\beta)=0$ 
In the literature, many techniques have been presented, to minimize the least squares norm $J$. Among these, the Newton, the conjugate gradients (Beck 1969; Orlande et al. 1999) and the Levenberg-Marquardt methods (Levenberg 1944; Marquardt 1963) are efficient for non-linear estimation.

Each algorithm has its advantages and its application domains. The Levenberg-Marquard algorithm has proved particular efficiency, because it presents a compromise between the Newton and the conjugate gradients method, therefore, we have chosen this method for the thermal proprieties estimation.

The iterative procedure to estimate the unknown parameters is given by:

$\beta^{k+1}=\beta^{k}+\Delta \beta^{k}$

Where $\Delta \beta^{k}$ is the increment of the unknown parameters vector at iteration $k$.

The increment $\Delta \beta^{k}$ for the Levenberg-Marquard method can be written as:

$\Delta \beta^{\mathrm{k}}=\left[\left(\mathrm{X}^{k}\right)^{T} \mathrm{X}^{k}+\mu^{k} \Omega^{k}\right]^{-1}\left(\mathrm{X}^{k}\right)^{T}\left[\mathrm{Y}-\mathrm{Y}_{\bmod }\left(\beta^{k}\right)\right]$

$X$ is the sensitivity matrix formed by the sensitivity coefficients at time $\mathrm{j}$ and point $\mathrm{i}$ to the unknown parameter $\beta_{\ell}$ given by:

$X_{i, \ell}^{j}=\frac{\Delta Y_{\bmod , i}^{j}}{\Delta \beta_{\ell}}$

where $i=1, \ldots, n ; \ell=1, \ldots, p$ and $j=1, \ldots, r$.

A new diagonal matrix term $\mu^{k} \Omega^{k}$ is added to $X^{T} X$ in order to damp oscillations and instabilities due to the ill-conditioned character of the problem. Where $\mu^{k}$ is a positive scalar named damping parameter, and $\Omega^{k}$ is a diagonal matrix (Levenberg 1944). In our case, we have considered $\Omega^{k}$ equal to the diagonal terms of $\left(X^{k}\right)^{T} X^{k}$.

\subsection{Sensitivity Analysis}

The sensitivity analysis of the temperature to the unknown parameters consists of analyzing the evolution of the different reduced sensitivity coefficients versus time. These reduced sensitivity coefficients are calculated using a forward finite difference approximation for $Y_{i}^{j}$ (Petit et al. 2005).

$$
\bar{X}^{j}{ }_{i, \ell}=\beta_{\ell} \cdot \frac{Y_{\bmod }{ }^{j}\left(\beta_{\ell}+\partial \beta_{\ell}\right)-Y_{\bmod }{ }^{j}\left(\beta_{\ell}\right)}{\Delta \beta_{\ell}}
$$

where $\partial \beta_{\ell}$ is taken equal to $10^{-3} \times \beta_{\ell}$ for each parameter. This common value of 0,001 was sufficient to approximate all the derivatives.

A careful investigation of the sensitivity coefficients often can aid in providing insight into the problem of determining parameters using nonlinear estimation. For this reason, $\bar{X}_{i, \ell}^{j}$ will be examined. One of the desired characteristics of a sensitivity coefficient in parameter estimation problems is that the magnitude of the coefficient be as large as possible. If there are two or more parameters to be found at one time, then in addition, the sensitivity coefficients should be as uncorrelated as possible (Jarni et al. 1995).

Thus, the sensitivity analysis permits to define the domain in which a variation of each parameter, independently to the others, generates a sensitive evolution in the studied system.

\subsection{Optimal Experiment Design}

The most common criteria for the optimal experiment design are based on the information matrix $X^{T} X$, which summarizes the information content of an experiment (Raynaud 1999). It is necessary to have a great determinant of $X^{T} X$ as a guaranty for great sensitivity coefficients and no correlated parameters. This D-criterion is presented as following:

Criteria $D=\operatorname{Arg}\left\{\max \operatorname{det}\left(\mathrm{X}^{T} \mathrm{X}\right)\right\}$

Another approach is to minimize the condition number of $X^{T} X$ that can be taken as the ratio of its largest eigenvalue to its smallest one. The more this value is near one; the more the problem is well posed.

Criteria $E=C N=\operatorname{Arg}\left\{\min \lambda_{\max } / \lambda_{\min }\right\}$

A compromise between these two approaches can be done; it consists in maximizing the lowest eigenvalue of $X^{T} X$. The physical motivation of this optimality is that it minimizes the largest parameter variance.

\section{RESUltS AND DISCUSSION}

In this study, we have considered a uniform rectangular metallic sample $\quad\left(\lambda=45 \quad \mathrm{~W} \cdot \mathrm{m}^{-1} \cdot \mathrm{K}^{-1}\right.$, $\left.\rho c_{p}=35,7374 \times 10^{5} \mathrm{~J} \cdot \mathrm{m}^{-3} \cdot \mathrm{K}^{-1}\right)$. The length, the width and the thickness of the plate, are, respectively $175 \mathrm{~mm}, 75$ $\mathrm{mm}$ and $0,65 \mathrm{~mm}$. The global heat transfer coefficient with the environment used in the simulations is $h=8$ $\mathrm{W} \cdot \mathrm{m}^{-2} \cdot \mathrm{K}^{-1}$.

The initial temperature of the medium is $\mathrm{T}_{0}=296,3 \mathrm{~K}$. An echelon of heat power with maximum value $\mathrm{S}=2,77 \times 10^{5} \mathrm{~W} \cdot \mathrm{m}^{-3}$ is injected within the volume of the plate.

For the sensitivity analysis, we have considered the sensor position $\mathrm{n}_{1}$ approximately near the middle ( $\mathrm{x}=85,25 \mathrm{~mm}$ and $\mathrm{y}=36,28 \mathrm{~mm}$ ), in order to analyze the behavior of the different reduced sensitivity coefficients versus time.

\subsection{Reduced Sensitivity Coefficients}

Considering the chosen position $\mathrm{n}_{1}$, we perform a sensitivity analysis of the temperature to the parameters $\left(\lambda, \rho c_{p}, h\right)$. Figure 2 shows that initially the reduced sensitivities, to the unknown parameters, vary in different manners, and then uncorrelated. 


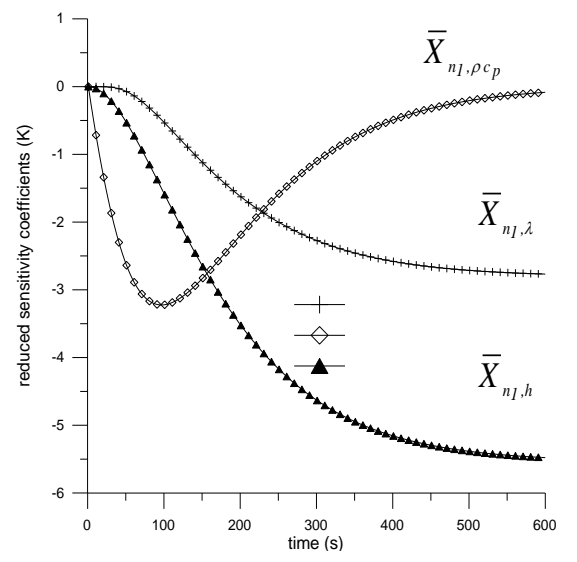

Fig. 2. Reduced sensitivity coefficients versus time

In fact, we notice that the thermal conductivity and the global heat transfer coefficient reach their maximums at the same time, but they decrease in different manners, so an eventual correlation can exist between these two parameters. This is clearly proved by the ratio of the reduced sensitivity coefficients (Fig. 3).

Therefore, the identification of the three parameters $\left(\lambda, \rho c_{p}, h\right)$ is feasible and the range time $[0,240 \mathrm{~s}]$ is sufficient to estimate simultaneously the thermal properties of this metallic medium.

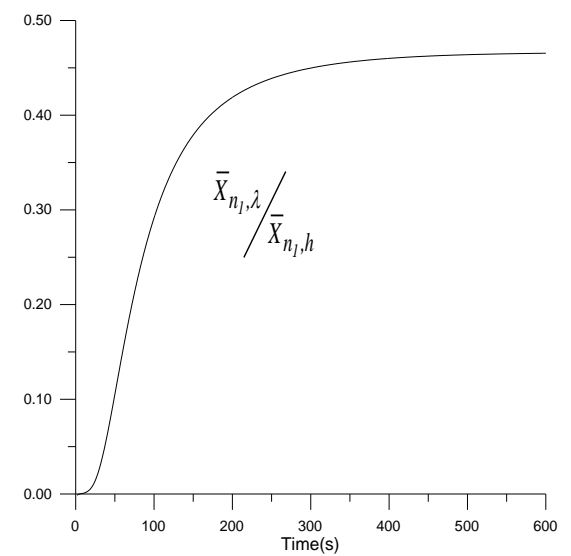

Fig. 3. Ratio of the reduced sensitivity coefficients

To perform an optimal experiment design, the influence of the sensors number on the determinant, the condition number and the lowest eigenvalue of the $X^{T} X$ is plotted in Fig. 4.

We see that the determinant and the lowest eingenvalue increase when the number $\mathrm{n}$ of the sensors increases. On the other hand, the condition number decreases with the increase of temperature measurements. Therefore, the parameters estimation might be best and will be less sensitive to the measurement errors while using many temperature sensors.

\subsection{Error Analysis}

Several types of errors can alter the validity of the information given by temperature measurements. To demonstrate the accuracy of the inverse method, the measured temperature is simulated. The input data
$Y_{\text {simulated }}($ Fig. 5) for the inverse problem is obtained by adding a noise term $\omega \sigma$ to the computed temperature $Y_{\text {mod }}$ (direct problem) as:

$Y_{\text {simulated }}=Y_{\text {mod }}+\omega \sigma$

The noise is Gaussian distributed and $\sigma$ is the standard deviation of the measurements errors. Assuming 99\% confidence for the measured data, $\omega$ lies in the range $-2,576 \leq \omega \leq 2,576$ and it is calculated by a random generator (Petit et al. 2005; Jarni et al. 1995).

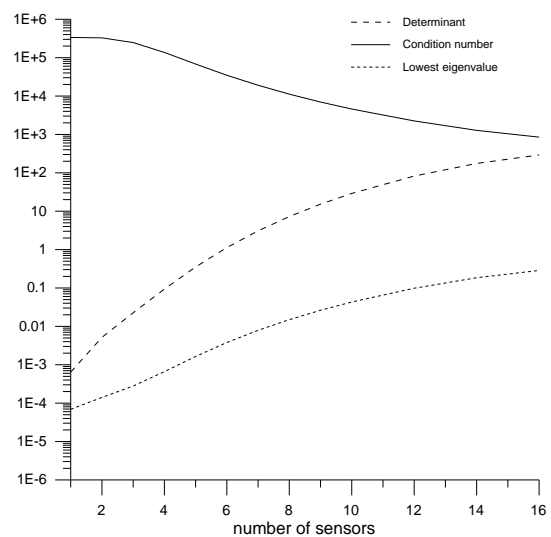

Fig. 4. Influence of sensors number on the determinant, the condition number and the lowest eigenvalue

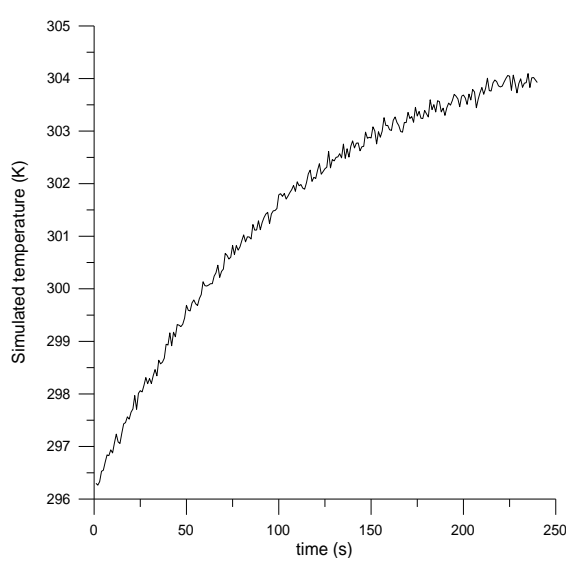

Fig. 5. Measured temperature at $\mathrm{n}_{1}$ versus time $(\sigma=0,1 \mathrm{~K})$

\subsection{Parameters Estimation}

Let us perform the identification of the thermal properties using simulated temperatures at position $n_{1}$. The results of the identification by the LevenbergMarquardt method for different standard deviations of measurement noise are shown in Table 1.

We report the identification results for initial values taken far away from the expected value.

We notice that, without measurement noise, the parameters are identified with a good accuracy. However, by the inclusion of temperature errors, the parameter identification becomes less accurate. On the other hand, the least squares norm $\mathrm{J}$ is very low $\left(10^{-20}\right)$ for standard deviation equal to zer $(\sigma=0 \mathrm{~K})$. But, when we add noise, the least squares increases. 
Table 1 Identification results using sensor position $\mathrm{n}_{1}$

\begin{tabular}{|c|c|c|c|c|}
\hline & $\begin{array}{c}\text { Initial } \\
\text { values }\end{array}$ & $\sigma=0 \mathrm{~K}$ & $\sigma=0,05 \mathrm{~K}$ & $\begin{array}{c}\sigma=0,1 \\
\mathrm{~K}\end{array}$ \\
\hline$\Lambda(45)$ & 65,0 & 44,999 & 46,471 & 50,731 \\
\hline $\begin{array}{c}\left(\rho \mathrm{C}_{\mathrm{p}}\right) \times 10^{5} \\
(35,7374)\end{array}$ & 20,0 & 35,7374 & 36,014 & 36,437 \\
\hline $\mathrm{H}(8)$ & 12,0 & 8,000 & 7,810 & 7,402 \\
\hline \multicolumn{2}{|c|}{$\begin{array}{c}\text { Number of } \\
\text { iterations }\end{array}$} & 8 & 2 & 3 \\
\hline \multicolumn{2}{|c|}{ Least squares J } & $8,7 \times 10^{-20}$ & 0,576 & 2,313 \\
\hline
\end{tabular}

An aspect of this parameter estimation problem is that the presence of measurements errors can influence the estimated values. In order to improve the quality of identification, we increased the number of measurement points. The identification results using 12 measurements intermediately between $\mathrm{x}_{1}$ and $\mathrm{x}_{12}$ positions as shown in Fig. 6, are reported in Table 2.

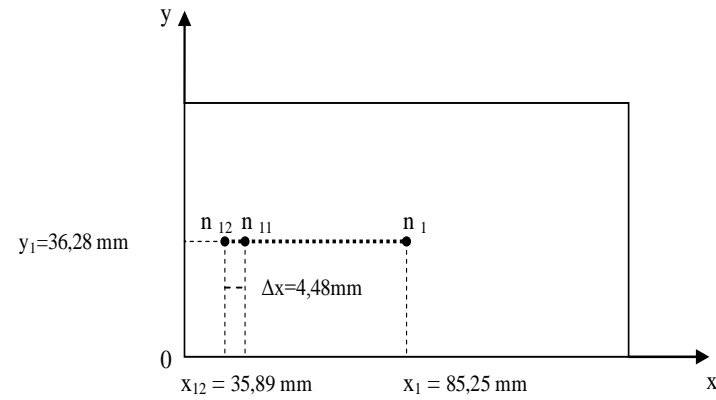

Fig. 6. Measurements temperatures positions

Table 2 Identification results using 12 temperatures measurements

\begin{tabular}{|c|c|c|c|c|}
\hline & $\begin{array}{c}\text { Initial } \\
\text { values }\end{array}$ & $\sigma=0 \mathrm{~K}$ & $\begin{array}{c}\sigma=0,05 \\
\mathrm{~K}\end{array}$ & $\begin{array}{c}\sigma=0,1 \\
\mathrm{~K}\end{array}$ \\
\hline$\Lambda(45)$ & 65,0 & 45,00 & 45,073 & 45,146 \\
\hline $\begin{array}{c}\left(\rho \mathrm{C}_{\mathrm{p}}\right) \times 10^{-5} \\
(35,7374)\end{array}$ & 20,0 & 35,7374 & 35,906 & 36,077 \\
\hline $\mathrm{h}(8)$ & 12,0 & 8,0001 & 7,958 & 7,917 \\
\hline \multicolumn{2}{|c|}{$\begin{array}{c}\text { Number of } \\
\text { iterations }\end{array}$} & 6 & 5 & 4 \\
\hline \multicolumn{2}{|c|}{ Least squares J } & $4,7 \times 10^{-18}$ & 6,955 & 27,821 \\
\hline
\end{tabular}

We notice that the previous results showed that while using many measurement points, the estimated parameters are in good agreement with the exact ones even when adding noise. For $\sigma=0$, the least squares $J$ is very low. However, when $\sigma \neq 0$, this criterion is "compatible" with the measurements noise. In fact,

$J \approx r \cdot n \cdot \sigma^{2}$

In our case, the number of the transient temperature $r$ is equal to 240 and the number of measurement sensors $n$ is equal to 12 .
Therefore, in this numerical study, the problem can be solved with the Levenberg-Marquardt method using many temperatures measurements. The parameters estimation will be less sensitive to the measurement errors while increasing the number of temperature measurements. This permits an accurate identification with a small number of iterations even when measurement noise is added and for initial guesses that are far from the expected values.

The proposed method can be applied to estimate simultaneously the effective thermal properties of the studied metallic medium, using infrared camera applied to the experimental set up built.

\section{EXPERIMENTAL SET-UP DESCRIPTION}

The main objective of the experimental Set-up conception, is to characterize the thermal behavior of metallic materials and then to determine thermal effects of iron losses.

The realization of the experimental installation imposes that the studied sample is easily visible, in order to be accessible to the infrared camera. A magnetic, an electric and a thermo-hydraulic circuit have been installed, so that, they do not embarrass the accessibility to the sample (Fig. 7).

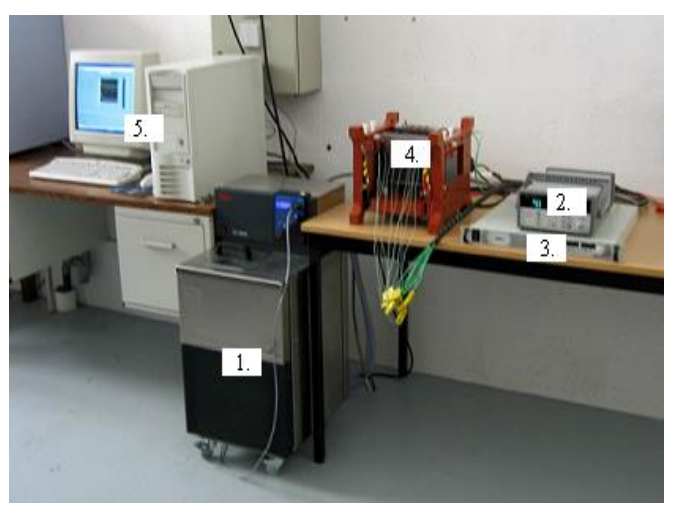

Fig. 7. The experimental set-up

This experimental set-up is made up of these parts:

1. Cryothermostat Huber CC 250 WL with a capacity tank until 5L. This cryothermostat can provide temperature ranged from $-40^{\circ} \mathrm{C}$ to $+200^{\circ} \mathrm{C}$. The power heating is limited to $1,2 \mathrm{~kW}$ at $20^{\circ} \mathrm{C}$, and $0,6 \mathrm{~kW}$ at $-20^{\circ} \mathrm{C}$.

2. Measurements central HP 34970A.

3. Power supply GEN 6-200 (0V-6V, 0A-200A)

4. the metallic sample and its support. This support can endure high temperature until $150^{\circ} \mathrm{C}$.

5. the controller computer

This Experimental Set-up permits to impose controlled magnetic and thermal constraints on the studied sample. The radiance due to the excitation system is blocked with two plates, cooled by a chimney effect. 
Two copper clips related to the hydraulic circuit, permit to have thermal boundary conditions (Fig. 8). These clips permit also the injection of a continuous current on the sample (Fig. 9). The electric power supplied to the sample can be measured; it is used to specify the dissipation in the plate, in order to identify thermal parameters.

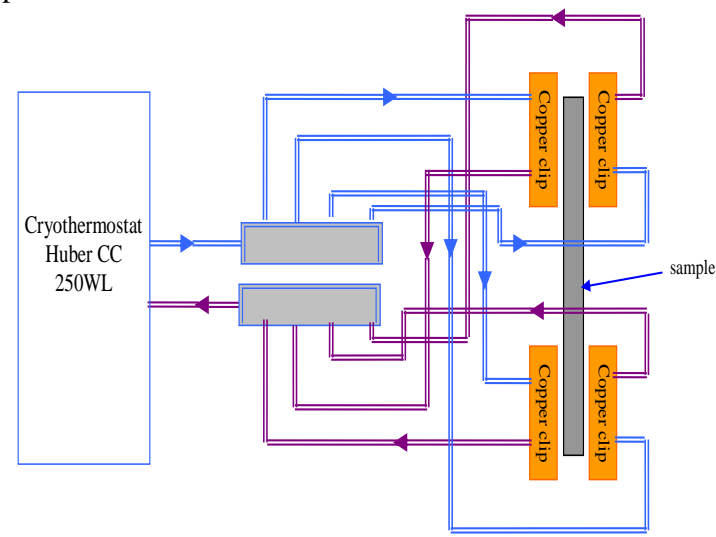

Fig. 8. The thermo-hydraulic circuit

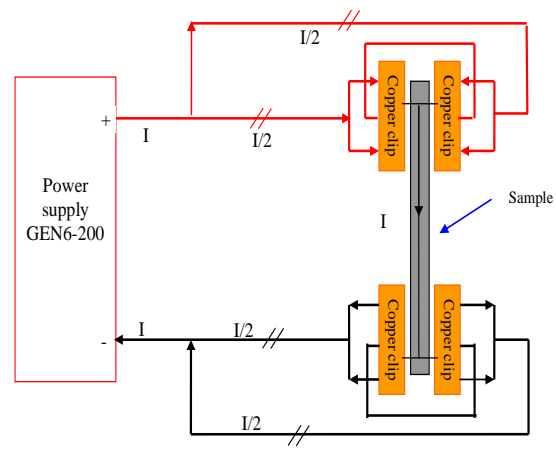

Fig. 9. The electric circuit

\section{EXPERIMENTAL VALIDATION}

The experimental set up was used to demonstrate the feasibility of the inversion and to characterize thermal properties of the studied medium.

The two clips permit to maintain the sample faces $(\mathrm{x}=0$ and $\mathrm{x}=\mathrm{L})$ to the ambient temperature, and to product an echelon of electric power in order to create a heat source in the plate volume $(\mathrm{P}=\mathrm{U} . \mathrm{I}=2,70 \mathrm{~W} ; \mathrm{I}=$ $68 \mathrm{~A}, \mathrm{U}=0,0398 \mathrm{~V})$.

By measuring the transient temperatures using an infrared camera, we represent, in title of illustration, temperatures in the plate (Fig. 10). Since the previous numerical study ( $\$ 4.3)$ have shown that the use of many temperature measurements permits an accurate identification with a small number of iterations, we proposed to use 12 temperatures measurements located intermediately between $n_{1}$ and $n_{12}$ positions as shown in Fig. 6.

The results of the experimental estimation performed with the same measurement positions us the simulated inversion, are given in Table 3 . We notice that the estimated values of the effective thermal conductivity,

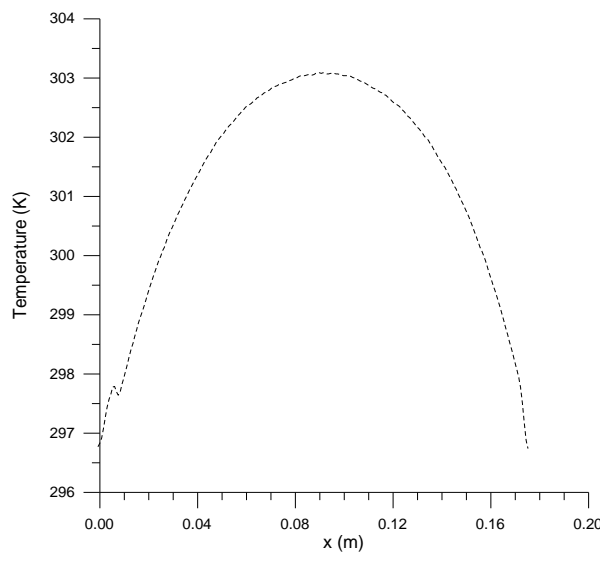

Fig. 10. Experimental measurements $(t=240 \mathrm{~s})$

the volumetric heat capacity and the global heat transfer coefficient with the environment have a good agreement with the thermal proprieties values of the magnetic steel $(\mathrm{Fe}-\mathrm{Se})$ provided by the literature "Verdun J" $\left(\lambda=42 \mathrm{~W} \cdot \mathrm{m}^{-1} \cdot \mathrm{K}^{-1}, \rho=7900 \mathrm{~kg} \cdot \mathrm{m}^{-3}, \mathrm{c}_{\mathrm{p}}=\right.$ $\left.490 \mathrm{~J}^{\mathrm{kg}} \mathrm{kg}^{-1} \cdot \mathrm{K}^{-1}\right)$. We notice that other estimations using various initial values were done and gave good results.

Table 3 Experimental estimation results

\begin{tabular}{|c|c|c|c|c|}
\hline & $\begin{array}{l}\text { Initial } \\
\text { values }\end{array}$ & $\begin{array}{l}\text { Estimated } \\
\text { values }\end{array}$ & $\begin{array}{l}\text { Number } \\
\text { of } \\
\text { iterations }\end{array}$ & $\begin{array}{l}\text { Least } \\
\text { squares } \\
\text { J }\end{array}$ \\
\hline$\lambda$ & 65,0 & 40,9 & \multirow{3}{*}{5} & \multirow{3}{*}{2,54} \\
\hline $\begin{array}{l}\left(\rho C_{p}\right) \\
\times 10^{-5} \\
\end{array}$ & 20,0 & 38,34 & & \\
\hline $\mathrm{h}$ & 7,0 & 10,24 & & \\
\hline
\end{tabular}

Figure 11 shows the transient measured temperatures and the calculated ones (model) using the estimated parameters $\left(\lambda=40,9 \mathrm{~W} \cdot \mathrm{m}^{-1} \cdot \mathrm{K}^{-1}, \rho c_{p}=38,34 \times 10^{5} \mathrm{~J} \cdot \mathrm{m}^{-}\right.$ ${ }^{3} \cdot \mathrm{K}^{-1}$ and $\left.h=10,24 \mathrm{~W} \cdot \mathrm{m}^{-2} \cdot \mathrm{K}^{-1}\right)$.

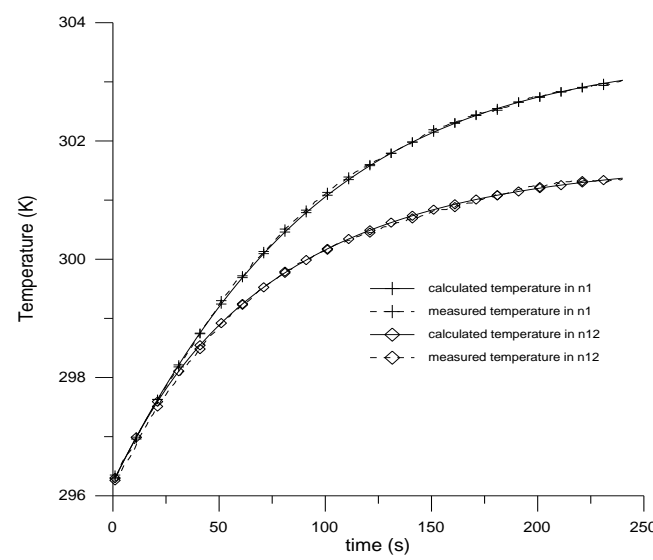

Fig. 11. Comparison of calculated and measured temperatures

We consider that the calculated temperatures approximate very closely the measured values. In order to attest the quality of the parameter estimation, we plot in Fig. 12 the evolution of the residues $r$ versus time "Maillet D", where: 


$$
r^{j}(\hat{\beta})=Y^{j}{ }_{\text {mes }}-Y_{\text {mod }}{ }^{j}(\hat{\beta})
$$

We notice that the residues are uncorrelated. This characteristic can attest of the quality of the parameters estimation and verify that the matrix sensibility $X$ is well conditioned.

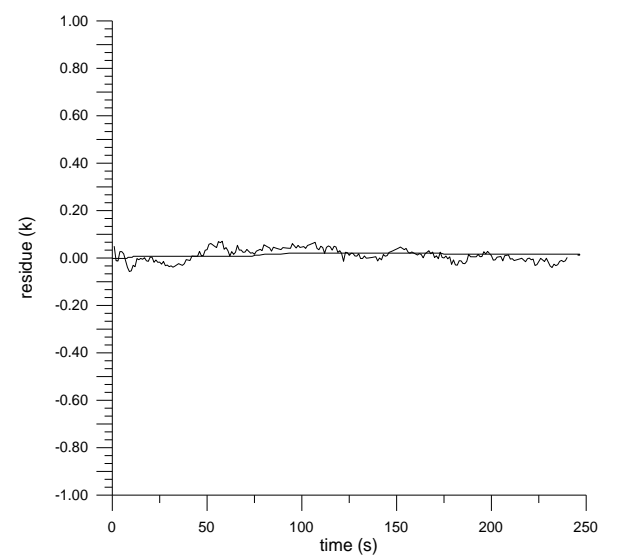

Fig. 12. Residue of estimation calculated at position $\mathrm{n}_{12}$

\section{CONCLUSION}

This paper provides a methodology to estimate the effective thermal properties in a metallic medium. A numerical study has been performed to identify, simultaneously, the effective thermal conductivity, the effective volumetric heat capacity and the global heat transfer coefficient with the environment. A sensitivity analysis has shown that the parameters are initially uncorrelated so, their simultaneously estimation is possible. In the case of the studied example, the Levenberg-Marquardt method appears to be efficient for the estimation. The use of the temperature evolution versus time measured at many positions permits an accurate estimation even with high measurements noise. The experimental validation of this study was done. The experimental set up built in the LET, permitted to demonstrate the feasibility of the estimation and verified that the proposed method for minimizing the least squares is valid.

An infrared camera is used to determine transient temperatures on the sample surface. The results showed that the simultaneously estimation of the thermal properties of the studied medium from temperature measurements is possible and is performed with a good accuracy.

\section{REFERENCES}

Amor, O. and M. Féliachi (1999). Magnetic hysteresis and its thermal behaviour in finite element computing. Electrimacs 99(3), 361-364.

Baliga, B.R. and S.V. Patankar (1983). A control volume finite-element method for two-dimensional fluid flow and heat transfer. Numerical Heat Transfer (6), 245-261.

Beck, J.V. (1969). Nonlinear estimation applied to the nonlinear inverse heat conduction problem. International Journal of Heat and Mass Transfer (13), 703-716.
Bertotti (1988). General properties of Power Losses in Soft Ferromagnetic Materials. IEEE Transanctions on Magnetics 24 (1).

Colaco, M.J. and H.R.B. Orlande (1999). Comparison of different versions of the conjugate gradient method of function estimation. Numerical of Heat Transfer A (36), 229-249.

Levenberg, K. (1944). A method for the solution of certain non-linear problems in least squares. Quarterly of Applied Mathematic (2), 164-168.

Marquardt, D.W. (1963). An algorithm for least squares estimation of nonlinear parameters. Journal of Social Industrial Applied Mathematic (11), 431441.

Petit, D. and D. Maillet (2005). Techniques inverses et estimation de paramètres, méthodologie et mise en œuvre. Technique de l'Ingénieur, (AF 4516).

Raynaud, M. (1999). Strategy for experimental design and the estimation of parameters. High Temperature-High Pressure (31), 1-15.

Sawaf, B., M.N. Ozisik and Y.Jarny (1995). An inverse analysis to estimate linearly temperature dependant thermal conductivity components and heat capacity of an orthotropic medium. International Journal of Heat and Mass Transfer 38 (16), 30053010.

Znaidia, S., F. Mzali, L. Sassi, A. Mhimid, A. Jemni, S.B. Nasrallah and D. Petit (2005). Inverse problem in a porous medium: estimation of the thermal properties. Inverse Problems in Science and Engineering 13 (6), 581-593. 